\title{
ESPECIAÇÃO REDOX DE CROMO EM SOLO ACIDENTALMENTE CONTAMINADO COM SOLUÇÃO SULFOCRÔMICA
}

\author{
Wladiana Oliveira Matos* e Joaquim de Araújo Nóbrega \\ Departamento de Química, Universidade Federal de São Carlos, Rod. Washington Luiz, km 235, 13565-905 São Carlos - SP, \\ Brasil \\ Gilberto Batista de Souza e Ana Rita Araujo Nogueira \\ Embrapa Pecuária Sudeste, CP 339, 13560-970 São Carlos - SP, Brasil
}

Recebido em 3/10/07; aceito em 15/2/08; publicado na web em 13/8/08

\begin{abstract}
CHROMIUM REDOX SPECIATION IN SOIL ACCIDENTALLY CONTAMINATED WITH SULPHOCHROMIC SOLUTION. Determination of $\mathrm{Cr}(\mathrm{VI})$ and $\mathrm{Cr}(\mathrm{III})$ was studied in soil samples accidentally contaminated with sulphochromic solution. Molecular absorption spectrophotometry based on the diphenylcarbazide method was used for the determination of Cr(VI) after its alkaline extraction. The total chromium concentration was determined using ICP OES. The quantification of Cr(III) was accomplished by subtracting the $\mathrm{Cr}(\mathrm{VI})$ concentration from the total chromium concentration. Regardless of the known contamination of the soil samples by sulphochromic solution, concentrations of $\mathrm{Cr}(\mathrm{VI})$ were below the detection limit. Addition and recovery experiments for $\mathrm{Cr}(\mathrm{VI})$ in soil samples with and without organic matter indicated its influence on the reduction of $\mathrm{Cr}(\mathrm{VI})$ to $\mathrm{Cr}(\mathrm{III})$.
\end{abstract}

Keywords: chromium; redox speciation; soil.

\section{INTRODUÇÃO}

O cromo apresenta diversas aplicações industriais incluindo seu emprego no processo de curtimento de couros, na preservação de madeira, como pigmentos, inibidor de corrosão etc. As principais indústrias que utilizam esse metal são das áreas de metalurgia, cerâmica e pigmentos. ${ }^{1}$

Outra intensa aplicação de compostos desse elemento é na determinação espectrofotométrica de matéria orgânica em amostras de solos, realizada especialmente em laboratórios de rotina. De acordo com a norma ISO 14235, Soil Quality - Determination of organic carbon by sulfochromic oxidation, estabelecida em 1998, a matéria orgânica pode ser determinada através de sua reação de oxidação com a mistura dicromato de potássio em excesso e ácido sulfúrico a uma temperatura de $135{ }^{\circ} \mathrm{C} .^{2}$

$$
2 \mathrm{Cr}_{2} \mathrm{O}_{7}^{2-}+3 \mathrm{C}+16 \mathrm{H}^{+} \rightarrow 4 \mathrm{Cr}^{3+}+3 \mathrm{CO}_{2}+8 \mathrm{H}_{2} \mathrm{O}_{(\mathrm{l})}
$$

Os íons dicromato, que apresentam coloração laranja, são reduzidos a $\mathrm{Cr}(\mathrm{III})$ de coloração verde e a intensidade deste último é medida espectrofotometricamente. Esse tipo de análise gera resíduos de íons cromato, ou seja, íons cromo no estado de oxidação VI que são potencialmente tóxicos ao ambiente. ${ }^{2} \mathrm{~A}$ análise de 2500 amostras de solo produz em torno de $150 \mathrm{~L}$ de solução ácida $(\mathrm{pH} \leq 1)$, contendo aproximadamente $10.000 \mathrm{mg} \mathrm{L}^{-1} \mathrm{Cr}(\mathrm{VI})$.

Embora o elemento cromo exista em diversos estados de oxidação, somente $\mathrm{Cr}(\mathrm{III})$ e $\mathrm{Cr}(\mathrm{VI})$ são suficientemente estáveis para ocorrer no ambiente. ${ }^{3}$ A presença de $\mathrm{Cr}(\mathrm{III})$ na dieta de animais e humanos é importante, pois está relacionada com o metabolismo de glicose, lipídeos e proteínas. ${ }^{4}$ Contudo, $\mathrm{Cr}(\mathrm{VI})$ é tóxico, carcinogênico e mutagênico para animais e humanos. ${ }^{5}$ Crômio(VI) apresenta mais mobilidade que $\mathrm{Cr}(\mathrm{III})$, pois seus ânions são facilmente transportados através do solo. ${ }^{6}$ Por outro lado, $\mathrm{Cr}(\mathrm{III})$ precipita como $\mathrm{Cr}(\mathrm{OH})_{3}$ ou $\mathrm{Fe}_{\mathrm{x}} \mathrm{Cr}_{1-\mathrm{x}}(\mathrm{OH})_{3}$ ou ainda forma quelatos com moléculas orgânicas,

\footnotetext{
*e-mail: wladianamatos@yahoo.com.br
}

tendo portanto menor mobilidade no solo. ${ }^{7,8}$

Pela toxicidade do $\mathrm{Cr}(\mathrm{VI})$ comparativamente ao $\mathrm{Cr}(\mathrm{III})$, a especiação redox de cromo se tornou importante, possibilitando um monitoramento ambiental bem mais adequado que apenas a quantificação do teor total do elemento. Devido à legislação estar se tornando cada vez mais restritiva, concentrações limites de $\mathrm{Cr}(\mathrm{VI})$ em diferentes matrizes sólidas estão sendo estabelecidas.

Na maioria dos solos o cromo é encontrado em concentrações que variam de 2 a $60 \mathrm{mg} \mathrm{kg}^{-1}$ dependendo do tipo de solo. ${ }^{9} \mathrm{Na}$ Suécia são sugeridos os valores de 5 e $120 \mathrm{mg} \mathrm{kg}^{-1}$ para $\mathrm{Cr}(\mathrm{VI})$ e $\mathrm{Cr}(\mathrm{III})$, respectivamente. ${ }^{10}$ No Canadá recomenda-se $0,4 \mathrm{mg} \mathrm{kg}^{-1} \mathrm{Cr}(\mathrm{VI})$ para solos destinados à agricultura e residencial e $1,4 \mathrm{mg} \mathrm{kg}^{-1}$ para solos comerciais e industriais. ${ }^{11}$ Já nos Estados Unidos a concentração limite de cromo pode diferir entre os estados. ${ }^{12} \mathrm{Na}$ Itália, a concentração máxima de $\mathrm{Cr}(\mathrm{VI})$ recomendada é de 2 a $15 \mathrm{mg} \mathrm{kg}^{-1}$ dependendo da ocupação do solo. ${ }^{13}$

Os métodos para tratamentos convencionais de solos e ambientes aquáticos contaminados com cromo hexavalente são a escavação ou bombeamento do material contaminado, adição de redutor químico, precipitação seguida de sedimentação, troca iônica e adsorção. ${ }^{14}$ Porém, $\mathrm{Cr}(\mathrm{VI})$ pode ser reduzido a $\mathrm{Cr}(\mathrm{III})$ no solo por reações redox com espécies inorgânicas, transferência de elétrons na superfície mineral, reações com substâncias orgânicas não húmicas, tais como carboidratos e proteínas, ou redução por substâncias húmicas do próprio solo..$^{15}$

Neste trabalho, foram avaliados os teores de $\mathrm{Cr}(\mathrm{III})$ e $\mathrm{Cr}(\mathrm{VI})$ em amostras de solo fornecidas pela Embrapa Agropecuária Oeste, localizada no município de Dourados no Mato Grosso do Sul, onde houve um vazamento de resíduos de solução sulfocrômica proveniente de um laboratório de solos.

\section{PARTE EXPERIMENTAL}

\section{Amostras}

As amostras de solo foram coletadas no município de Dourados - MS, na sede da Embrapa Agropecuária Oeste. Nessa unidade da 
Embrapa, assim como em outras, o laboratório de solos utiliza o método ISO 14235 para a determinação de matéria orgânica no solo e os resíduos de íons cromato gerados pelas análises são armazenados em reservatórios para posterior tratamento adequado. No caso particular estudado, esse reservatório se rompeu, causando contaminação por $\mathrm{Cr}(\mathrm{VI})$ do solo localizado nas proximidades do reservatório. Com o objetivo de avaliar a gravidade do prejuízo ambiental causado pelo acidente, amostras desse solo foram enviados à Embrapa Pecuária Sudeste (São Carlos-SP) para que estudos de especiação redox de cromo fossem realizados.

Material de referência certificado de solo NIST San Joaquin Soil SRM 2709, EUA foi utilizado para verificar a exatidão da determinação de cromo total em solo.

\section{Reagentes e soluções}

Todos os materiais utilizados no trabalho foram descontaminados em banho de $\mathrm{HCl} \mathrm{10 \%} \mathrm{(v/v)} \mathrm{por} \mathrm{no} \mathrm{mínimo} 24$ h, lavados com água destilada e com água ultrapura (resistividade de $18,2 \mathrm{M} \Omega \mathrm{cm}$ ) obtida a partir de um sistema de purificação de água Milli-Q (Millipore, Bedford, MA, EUA).

Para todos os experimentos, reagentes de grau analítico e água ultrapura foram empregados para preparo de solução.

Solução padrão estoque $1000 \mathrm{mg} \mathrm{L}^{-1} \mathrm{Cr}(\mathrm{VI})$ foi preparada dissolvendo-se 2,830 g de $\mathrm{K}_{2} \mathrm{Cr}_{2} \mathrm{O}_{7}$ (Riedel-de Haën, Alemanha), previamente seco à temperatura de $140{ }^{\circ} \mathrm{C}$, em $1 \mathrm{~L}$ de água. Solução padrão estoque $1000 \mathrm{mg} \mathrm{L}^{-1} \mathrm{Cr}$ (III) (Merck, Alemanha) foi diluída para preparo das soluções analíticas de calibração.

Os ácidos clorídrico 36\% (v/v), nítrico 65\% (v/v) e fluorídrico $36 \%$ (v/v) utilizados para a decomposição total das amostras estudadas foram obtidos da Merck. Os reagentes peróxido de hidrogênio $30 \%(\mathrm{~m} / \mathrm{m}), 1,5$-difenilcarbazida, carbonato de sódio e ácido bórico também tinham a mesma procedência. A acetona empregada no preparo das soluções de 1,5-difenilcarbazida foi produzida pela Synth (Brasil) e o ácido sulfúrico concentrado para ajuste de $\mathrm{pH}$ e preparo da solução de 1,5-difenilcarbazida foi produzido pela Mallinckrodt (Cidade do México, México).

\section{Extração e determinação de $\operatorname{Cr}(\mathrm{VI})$}

A extração de $\mathrm{Cr}(\mathrm{VI})$ nas amostras sólidas foi efetuada empregando solução alcalina sob aquecimento. O meio alcalino foi escolhido por minimizar os riscos de redução dos íons $\mathrm{Cr}(\mathrm{VI})$ a $\mathrm{Cr}(\mathrm{III})$. A solução $0,10 \mathrm{~mol} \mathrm{~L}^{-1} \mathrm{Na}_{2} \mathrm{CO}_{3}$ foi utilizada como extrator. Essa solução extratora foi empregada por Panichev et al. ${ }^{16}$ para extração de $\mathrm{Cr}(\mathrm{VI})$ em amostras de solo.

A solução $0,10 \mathrm{~mol} \mathrm{~L}^{-1} \mathrm{Na}_{2} \mathrm{CO}_{3}$ foi preparada pesando-se 1,06 $\mathrm{g}$ de carbonato de sódio e dissolvendo-se o sal com água para um volume de $100 \mathrm{~mL}$. O volume do extrator utilizado no procedimento de extração de $\mathrm{Cr}(\mathrm{VI})$ variou de acordo com o tipo de amostra. A extração de $\mathrm{Cr}(\mathrm{VI})$ foi realizada sob aquecimento em banho de areia por período de 10 min, contado a partir do momento da ebulição da solução.

As determinações de $\mathrm{Cr}(\mathrm{VI})$ em todas as amostras foram efetuadas por espectrofotometria de absorção molecular na região do visível empregando 1,5-difenilcarbazida como reagente cromogênico. De acordo com esse método, $\mathrm{Cr}(\mathrm{VI})$ presente na solução reage com solução de 1,5-difenilcarbazida em meio ácido ( $\mathrm{pH} 1$ ) produzindo uma solução violeta. Esse é um método sensível e seletivo para a determinação espectrofotométrica de $\mathrm{Cr}(\mathrm{VI}) .{ }^{17}$

Para preparar a solução de 1,5-difenilcarbazida, 0,2 g desse reagente foi pesado e dissolvido em $100 \mathrm{~mL}$ de acetona contendo 1 $\mathrm{mL}$ de $\mathrm{H}_{2} \mathrm{SO}_{4}(1+9(\mathrm{v} / \mathrm{v}))$. A solução foi transferida e mantida em frasco âmbar para evitar degradação fotoquímica do reagente. As soluções de 1,5-difenilcarbazida utilizadas no experimento foram refeitas semanalmente.

\section{Preparo de amostra para quantificação do teor total de cromo}

Forno de microondas com cavidade utilizando frascos fechados e ácidos concentrados foi empregado para a digestão buscando a decomposição completa das amostras para viabilizar as determinações por ICP OES. Após digestão, foi adicionado ácido fluorídrico à temperatura ambiente para solubilizar os silicatos. O procedimento proposto por Vieira et al. ${ }^{18}$ envolve apenas um ciclo de digestão evitando, assim, desgaste dos frascos reacionais e excessivo consumo de tempo para o preparo das amostras. Além disso, evita-se a formação de sais insolúveis de fluoreto observada quando a adição de ácido fluorídrico ocorre no início do processo de digestão ou após a decomposição, permitindo a determinação elementar com exatidão e precisão para diferentes tipos de solo.

\section{Instrumentação}

Espectrômetro de emissão óptica com plasma acoplado indutivamente (ICP OES) com visão radial (Vista PRO-CCD, Varian, Austrália) foi utilizado para as determinações de cromo total. Aplicou-se potência de $1,3 \mathrm{~kW}$ e vazão de nebulização de $0,6 \mathrm{~L} \mathrm{~min}^{-1}$. Utilizou-se sistema de introdução de amostra com nebulizador com ranhura em V e câmara de nebulização Sturman-Master. A altura de observação foi de $6 \mathrm{~mm}$ e o comprimento de onda de 206,158 nm foi escolhido para as determinações de cromo.

Espectrofotômetro de absorção molecular (UV-Vis modelo 482, Femto, São Paulo) foi utilizado para as determinações de Cr(VI), após reação desse íon com solução de 1,5-difenilcarbazida. O comprimento de onda empregado nas determinações de $\mathrm{Cr}(\mathrm{VI})$ foi de $545 \mathrm{~nm}$.

Forno com cavidade Multiwave ${ }^{\circledR}$ (Anton Paar Graz, Áustria) com frascos de alta pressão de TFM com volume de $50 \mathrm{~mL}$ foi utilizado para a decomposição completa das amostras de solo. $\mathrm{O}$ aquecimento da solução para as extrações de $\mathrm{Cr}(\mathrm{VI})$ foi feito em banho de areia.

Os digeridos de solo foram centrifugados utilizando centrífuga Hermele Z modelo 200 A (Labnet, Woodbridge, NJ, EUA).

O procedimento de calcinação das amostras de solo foi efetuado em mufla usando cadinho de porcelana.

\section{Procedimento experimental}

\section{Teste de estabilidade do Cr(VI)}

Foi avaliado o comportamento redox do $\mathrm{Cr}(\mathrm{VI})$ na solução extratora $0,10 \mathrm{~mol} \mathrm{~L}^{-1} \mathrm{Na}_{2} \mathrm{CO}_{3}$ com o objetivo de testar a estabilidade desse ânion. Os experimentos foram realizados em triplicata.

Solução contendo $12,5 \mathrm{mg} \mathrm{L}^{-1} \mathrm{Cr}(\mathrm{VI})$ foi preparada a partir da solução padrão estoque de $1000 \mathrm{mg} \mathrm{L}^{-1} \mathrm{Cr}(\mathrm{VI})$. Uma alíquota de 5 $\mathrm{mL}$ da solução com concentração $12,5 \mathrm{mg} \mathrm{L}^{-1} \mathrm{Cr}(\mathrm{VI})$ foi adicionada a $25 \mathrm{~mL}$ da solução $0,10 \mathrm{~mol} \mathrm{~L}^{-1} \mathrm{Na}_{2} \mathrm{CO}_{3}$. A mistura foi aquecida em um banho de areia por $30 \mathrm{~min}$, de maneira a simular o procedimento adotado para extração em uma amostra. Após a etapa de aquecimento, a solução foi resfriada à temperatura ambiente, transferida para um tubo graduado de $50 \mathrm{~mL}$ e o volume foi ajustado para $25 \mathrm{~mL}$ com água. Em seguida, uma alíquota de $2 \mathrm{~mL}$ dessa solução foi retirada para quantificação de $\mathrm{Cr}(\mathrm{VI})$. Dessa forma, adicionou-se solução $0,1 \mathrm{~mol} \mathrm{~L}^{-1} \mathrm{H}_{2} \mathrm{SO}_{4}$ em tubo graduado de $50 \mathrm{~mL}$ visando ajustar o $\mathrm{pH}$ para 1, sendo, em seguida, adicionado $1 \mathrm{~mL}$ de 1,5-difenilcarbazida. $\mathrm{O}$ volume final foi ajustado para $25 \mathrm{~mL}$ com solução $0,1 \mathrm{~mol} \mathrm{~L}^{-1}$ $\mathrm{H}_{2} \mathrm{SO}_{4}$ e a absorbância da solução resultante, de coloração violeta, foi medida. 
Esse mesmo experimento foi repetido, porém, além da solução padrão de $\mathrm{Cr}(\mathrm{VI})$, solução padrão de $\mathrm{Cr}$ (III) também foi adicionada com o intuito de avaliar o comportamento do cromo hexavalente na presença de cromo trivalente, observando-se as porcentagens de recuperação de $\mathrm{Cr}(\mathrm{VI})$. Assim, soluções de $12,5 \mathrm{mg} \mathrm{L}^{-1} \mathrm{Cr}(\mathrm{III})$ e $\mathrm{Cr}(\mathrm{VI})$ foram preparadas a partir das soluções padrão estoque $1000 \mathrm{mg} \mathrm{L}^{-1}$ $\mathrm{Cr}(\mathrm{III})$ e $\mathrm{Cr}(\mathrm{VI})$. Alíquotas de $5 \mathrm{~mL}$ de solução padrão $12,5 \mathrm{mg} \mathrm{L}^{-1}$ de ambos estados de oxidação foram adicionadas a $25 \mathrm{~mL}$ da solução $0,10 \mathrm{~mol} \mathrm{~L}^{-1} \mathrm{Na}_{2} \mathrm{CO}_{3}$. O procedimento adotado foi idêntico ao descrito no parágrafo anterior para as soluções contendo apenas $\mathrm{Cr}(\mathrm{VI})$.

\section{Preparo de amostra de Cr total ${ }^{18}$}

Na digestão das amostras de solo, pesou-se $100 \mathrm{mg}$ de amostra em frascos de digestão e adicionou-se um volume de $2 \mathrm{~mL}$ de água régia (ácido nítrico e ácido clorídrico 1:3 (v/v)) e $1 \mathrm{~mL}$ de $\mathrm{H}_{2} \mathrm{O}_{2}$ concentrado. Essa mistura foi submetida a um programa de aquecimento com tempo total de 34 min (Tabela 1). A decomposição foi conduzida em frascos fechados com aquecimento assistido por radiação microondas. Após resfriamento, os digeridos foram quantitativamente transferidos para frascos graduados com volume de $15 \mathrm{~mL}$, sendo o volume ajustado para $10 \mathrm{~mL}$. A fase insolúvel contendo silicatos não digeridos foi separada por centrifugação (3 min, $2000 \mathrm{rpm}$ ). O sobrenadante foi transferido para outro tubo de $15 \mathrm{~mL}$ e o precipitado foi dissolvido à temperatura ambiente adicionando-se $1 \mathrm{~mL}$ de $\mathrm{HF}$ concentrado. Após dissolução dos silicatos, adicionou-se $500 \mathrm{mg}$ de $\mathrm{H}_{3} \mathrm{BO}_{3}$ para complexação dos fluoretos remanescentes. A mistura resultante foi acrescentada ao sobrenadante recolhido anteriormente e o volume foi ajustado para $15 \mathrm{~mL}$ com água.

Tabela 1. Programa de aquecimento utilizado na decomposição de amostra de solo em forno de microondas com cavidade (Anton Paar). Temperatura máxima de trabalho: $210^{\circ} \mathrm{C}$

\begin{tabular}{lccccc}
\hline Etapa & $\begin{array}{c}\text { Potência } \\
\text { inicial (W) }\end{array}$ & $\begin{array}{c}\text { Tempo } \\
(\mathrm{min})\end{array}$ & $\begin{array}{c}\text { Potência } \\
\text { final (W) }\end{array}$ & $\begin{array}{c}\text { Ventilação } \\
(\%)\end{array}$ & Descrição \\
\hline 1 & 400 & 3,0 & 400 & 25 & $\begin{array}{c}\text { Pré-aquec- } \\
\text { imento } \\
2\end{array}$ \\
3 & 1000 & 10,0 & 1000 & 25 & $\begin{array}{c}\text { Aqueci- } \\
\text { mento } \\
\text { Aqueci- } \\
\text { mento } \\
\text { Resfria- } \\
\text { mento }\end{array}$ \\
\hline
\end{tabular}

\section{Preparo de amostra e determinação de Cr(VI)}

No procedimento de extração de $\mathrm{Cr}(\mathrm{VI})$ das amostras de solo, $250 \mathrm{mg}$ de solo foram pesados em béquer de vidro e a essa massa foi adicionado $25 \mathrm{~mL}$ de solução extratora $0,1 \mathrm{~mol} \mathrm{~L}^{-1} \mathrm{Na}_{2} \mathrm{CO}_{3}$. A mistura foi mantida sob ebulição em banho de areia por $10 \mathrm{~min}$, tempo suficiente para completa extração de $\mathrm{Cr}(\mathrm{VI})$ empregando solução 0,1 mol L ${ }^{-1} \mathrm{Na}_{2} \mathrm{CO}_{3}$, segundo Panichev et al.. ${ }^{16}$ Após extração, a solução resultante foi resfriada à temperatura ambiente e transferida para tubo graduado de $50 \mathrm{~mL}$, sendo o volume ajustado para $25 \mathrm{~mL}$. A solução foi centrifugada por $10 \mathrm{~min}$ (4000 rpm). Um volume de 10 $\mathrm{mL}$ do sobrenadante foi retirado e transferido para tubo graduado de $50 \mathrm{~mL}$ para reação com 1,5-difenilcarbazida. Para isso, o pH da solução foi ajustado para $1 \mathrm{com}$ a utilização de $\mathrm{H}_{2} \mathrm{SO}_{4} 5 \mathrm{~mol} \mathrm{~L}^{-1}$. Em seguida, $1 \mathrm{~mL}$ da solução de 1,5-difenilcarbazida foi acrescentado e, após desenvolvimento da reação, a absorbância da solução colorida resultante foi medida. Como o extrato apresentou ligeira coloração, sinais de absorção para brancos analíticos contendo as amostras e todos os reagentes empregados no procedimento de extração, porém sem adição do reagente cromogênico, foram medidos para cada amostra. A absorbância do branco analítico foi subtraída do sinal de absorbância da respectiva amostra. A curva analítica foi construída usando soluções de referência preparadas a partir do branco analítico. Os experimentos foram realizados em triplicata.

\section{Adição e recuperação de Cr(VI) em amostra de solo calcinado e não calcinado}

Procedimento de calcinação de amostra de solo foi aplicado para verificar a influência da matéria orgânica sobre a determinação de $\mathrm{Cr}(\mathrm{VI})$. Nesse procedimento, duas alíquotas de $55 \mathrm{~g}$ da mesma amostra de solo foram pesadas. Uma delas foi colocada em cadinho de porcelana e aquecida em forno tipo mufla à temperatura de $500^{\circ} \mathrm{C}$ por $10 \mathrm{~h}$ a fim de que toda a matéria orgânica fosse eliminada. Após resfriamento da amostra que foi submetida à calcinação, adicionaramse volumes de $14 \mathrm{~mL}$ de solução padrão $1000 \mathrm{mg} \mathrm{L}^{-1} \mathrm{Cr}(\mathrm{VI})$ em ambas alíquotas de solo. As misturas foram homogeneizadas utilizando almofariz e pistilo e aquecidas em estufa à temperatura de $30^{\circ} \mathrm{C}$ até a secura. Em seguida, foram realizadas determinações de matéria orgânica pelo método ISO 14235 e quantificação de $\mathrm{Cr}$ (VI) com o método da 1,5-difenilcarbazida nas duas alíquotas da amostra de solo.

\section{RESULTADOS E DISCUSSÃO}

\section{Estabilidade do íon $\mathrm{Cr}(\mathrm{VI})$}

A avaliação da estabilidade da forma redox que se deseja quantificar é de fundamental importância na análise de especiação. No caso da especiação redox de cromo, espécies de $\mathrm{Cr}(\mathrm{VI})$ podem ser reduzidas à forma $\mathrm{Cr}(\mathrm{III})$, resultando em teores de $\mathrm{Cr}(\mathrm{VI})$ inferiores ao conteúdo original. Ou ainda, íons $\mathrm{Cr}(\mathrm{III})$ presentes no meio podem ser oxidados a $\mathrm{Cr}(\mathrm{VI})$ e, conseqüentemente, os teores de $\mathrm{Cr}(\mathrm{VI})$ determinados serão afetados por erros positivos.

Assim, experimentos de adição e recuperação de $\mathrm{Cr}(\mathrm{VI})$ na presença e na ausência de íons $\mathrm{Cr}(\mathrm{III})$ foram realizados usando solução extratora $0,10 \mathrm{~mol} \mathrm{~L}^{-1} \mathrm{Na}_{2} \mathrm{CO}_{3}$. No primeiro experimento, apenas $\mathrm{Cr}(\mathrm{VI})$ na concentração final de $2,0 \mathrm{mg} \mathrm{L}^{-1}$ estava presente na solução extratora. No segundo, a solução extratora continha quantidades equivalentes a $1,8 \mathrm{mg} \mathrm{L}^{-1} \mathrm{Cr}(\mathrm{VI})$ e $1,8 \mathrm{mg} \mathrm{L}^{-1} \mathrm{Cr}(\mathrm{III})$. Essas misturas foram submetidas a aquecimento em banho de areia por $30 \mathrm{~min}$, visando simular o procedimento de extração aplicado para amostras.

A recuperação em porcentagem de $\mathrm{Cr}(\mathrm{VI})$ quando se adicionou apenas padrão de $\mathrm{Cr}(\mathrm{VI})$ foi de $101,9 \pm 2,8$ e com adição da mistura das espécies $\mathrm{Cr}(\mathrm{VI})$ e $\mathrm{Cr}(\mathrm{III})$ foi de $109,1 \pm 2,3$. Os resultados apresentam valores médios e os respectivos desvios padrão $\operatorname{com} n=3$.

Os dados dos testes indicam que $0,10 \mathrm{~mol} \mathrm{~L}^{-1} \mathrm{Na}_{2} \mathrm{CO}_{3}$ com as condições empregadas no procedimento de preparo de amostra, isto é, aquecimento em banho de areia por $30 \mathrm{~min}$, não causou significativa transformação redox do íon $\mathrm{Cr}(\mathrm{VI})$, tanto na ausência quanto na presença de íons $\mathrm{Cr}(\mathrm{III})$, pois valores de recuperações de Cr(VI) equivalentes a 101,9 e 109,1\% foram obtidos. Dessa forma, confirma-se a exatidão proporcionada por essa solução alcalina para as extrações de $\mathrm{Cr}(\mathrm{VI})$ na análise de especiação redox de cromo em amostras sólidas. Esses resultados estão de acordo com a literatura, compostos de $\mathrm{Cr}(\mathrm{VI})$ em soluções ácidas têm forte tendência a se reduzirem. Em contrapartida, compostos de $\mathrm{Cr}(\mathrm{VI})$ têm maior estabilidade em meios com pH's mais elevados. ${ }^{19}$

\section{Especiação redox de cromo em amostras de solo}

A presença de $\mathrm{Cr}(\mathrm{VI})$ em solos é preocupante, pois compostos desse íon são, geralmente, solúveis implicando em maior mobilidade no ambiente. Dessa forma, contaminações de reservatórios de águas devido 
à lixiviação do $\mathrm{Cr}(\mathrm{VI})$ presente no solo por chuvas podem ocorrer.

As amostras de solo analisadas haviam sido acidentalmente contaminadas com solução sulfocrômica residual proveniente de determinação de matéria orgânica em solos empregando método ISO 14235. Essa solução normalmente contém aproximadamente 10000 $\mathrm{mg} \mathrm{L}^{-1}$ de $\mathrm{Cr}(\mathrm{VI})$, conforme determinações realizadas anteriormente (dados não publicados). Em função disso, esperava-se obter altas concentrações de $\mathrm{Cr}(\mathrm{VI})$ nas amostras de solo analisadas, contudo não foi o que ocorreu (Tabela 2).

Tabela 2. Especiação redox de cromo nas amostras de solo contaminado com solução sulfocrômica

\begin{tabular}{lcc}
\hline Amostra & ${ }^{1} \mathrm{Cr}(\mathrm{VI})\left(\mathrm{mg} \mathrm{kg}^{-1}\right)$ & $\mathrm{Cr}$ total $\left(\mathrm{mg} \mathrm{kg}^{-1}\right)$ \\
\hline $1 \mathrm{a}$ & $<0,01$ & $162,9 \pm 3,0$ \\
$1 \mathrm{~b}$ & $<0,01$ & $98,6 \pm 1,8$ \\
$1 \mathrm{c}$ & $<0,01$ & $148,7 \pm 3,6$ \\
2 & $<0,01$ & $190,9 \pm 10,1$ \\
3 & $<0,01$ & $152,5 \pm 4,0$ \\
4 & $<0,01$ & $92,1 \pm 1,1$ \\
\hline
\end{tabular}

${ }^{1}$ LOD: $0,01 \mathrm{mg} \mathrm{kg}^{-1}$; 1a: 0-20 cm; $1 \mathrm{~b}: 20-40 \mathrm{~cm}$ e 1c: $40-60 \mathrm{~cm} \mathrm{de}$ profundidade de coleta. Os resultados representam valores médios e os respectivos desvios padrão $(\mathrm{n}=3)$

O teor de $\mathrm{Cr}(\mathrm{VI})$ ficou abaixo do limite de detecção (LOD = 0,01 $m g \mathrm{~kg}^{-1} \mathrm{de} \mathrm{Cr}(\mathrm{VI})$ ), sendo o teor de $\mathrm{Cr}(\mathrm{III})$ equivalente à concentração de cromo total obtida na determinação por ICP OES.

A CETESB (Companhia de Tecnologia de Saneamento Ambiental), através da Decisão de Diretoria № 195-2005-E de novembro de 2005, dispõe valores orientadores para solos e águas subterrâneas no estado de São Paulo. Os valores orientadores são concentrações de substâncias químicas que definem a condição de qualidade de solo e água subterrânea e são utilizados como prevenção e controle da contaminação e gerenciamento de áreas contaminadas sob investigação. Com relação ao elemento cromo no solo, teores acima de 75 $\mathrm{mg} \mathrm{kg}^{-1}$ são designados como valores de prevenção, ou seja, nesse caso podem ocorrer alterações prejudiciais à qualidade do solo. Concentrações de 150, 300 e $400 \mathrm{mg} \mathrm{kg}^{-1}$ indicam solo com valor de intervenção para cenários de exposição Agrícola-Área de Proteção Máxima, Residencial e Industrial, respectivamente, exibindo riscos potenciais, diretos ou indiretos, à saúde humana. ${ }^{20}$ As concentrações de cromo total, apresentadas na Tabela 2, variaram entre 92,1 e 190,9 $\mathrm{mg} \mathrm{kg}^{-1}$, o que enquadra os resultados das amostras de solo analisadas em ambos os casos, valores de prevenção e intervenção.

Material de referência certificado de solo com concentração 130 $\pm 4 \mathrm{mg} \mathrm{kg}^{-1}$ de cromo foi também analisado para verificar a exatidão da quantificação do teor total do elemento, obtendo-se um teor de $129,6 \pm 4,1 \mathrm{mg} \mathrm{kg}^{-1}$ (média \pm desvio padrão, $\mathrm{n}=3$ ). O percentual de recuperação, portanto, foi de $99,7 \%$, o que indica a adequada exatidão do procedimento de determinação de cromo total.

Como nas amostras de solo havia ocorrido um derramamento do reservatório de solução sulfocrômica residual e as análises de especiação redox de cromo apresentaram concentrações de $\mathrm{Cr}(\mathrm{VI})$ abaixo do limite de detecção, a redução de $\mathrm{Cr}(\mathrm{VI})$ deve ter ocorrido devido à presença de composto facilmente oxidável nos solos analisados. Alguns trabalhos na literatura afirmam que $\mathrm{Cr}(\mathrm{VI})$ em solo pode ser reduzido a $\mathrm{Cr}(\mathrm{III})$, especialmente em solos contendo altos teores de matéria orgânica. Esse processo de redução é ainda mais acentuado quando se tem solos com $\mathrm{pH}$ ácido. ${ }^{21-23}$ Para elucidar os resultados obtidos e considerando-se um possível processo redox entre $\mathrm{Cr}(\mathrm{VI})$ e matéria orgânica, determinaram-se os teores de matéria orgânica nas amostras de solo contaminadas (Tabela 3 ).
Tabela 3. Teores de matéria orgânica nas amostras de solo

\begin{tabular}{lc}
\hline Amostra & M. O. $\left(\mathrm{g} \mathrm{kg}^{-1}\right)$ \\
\hline $1 \mathrm{a}$ & $32,0 \pm 0,8$ \\
$1 \mathrm{~b}$ & $27,0 \pm 0,6$ \\
$1 \mathrm{c}$ & $18,0 \pm 0,8$ \\
2 & $30,0 \pm 0,8$ \\
3 & $28,0 \pm 0,4$ \\
4 & $24,0 \pm 0,3$ \\
\hline
\end{tabular}

1a: $0-20 \mathrm{~cm}$; $1 \mathrm{~b}: 20-40 \mathrm{~cm}$ e $1 \mathrm{c}$ : $40-60 \mathrm{~cm}$ de profundidade de coleta. Os resultados representam valores médios e os respectivos desvios padrão $(\mathrm{n}=3)$.

Os dados da Tabela 3 indicam a presença de matéria orgânica em todas as amostras de solo, com teores que variam de 18,0 a 32,0 $\mathrm{g} \mathrm{kg}^{-1}$. Esses resultados confirmaram a presença de agentes redutores. Dessa forma, concentrações significativas de $\mathrm{Cr}(\mathrm{VI})$ provavelmente estavam presentes originalmente nas amostras de solo contaminadas com solução sulfocrômica residual. Contudo, os intervalos de tempo entre o processo de contaminação, etapa de coleta e etapa de determinação foram suficientemente longos para que processos redox envolvendo $\mathrm{Cr}(\mathrm{VI})$ e matéria orgânica ocorressem e impedissem a detecção do $\mathrm{Cr}(\mathrm{VI})$ proveniente do processo de contaminação. Pode-se supor que a fração majoritária do $\mathrm{Cr}(\mathrm{III})$ detectado foi gerada por esse processo redox e decorre, portanto, do processo de contaminação original. Assim, a contaminação de um solo por $\mathrm{Cr}(\mathrm{VI})$ seria mais drástica para ambientes com baixos teores de matéria orgânica, pois nesse caso o cromo permaneceria na forma de $\mathrm{Cr}(\mathrm{VI})$, tóxica e indesejável no ambiente.

Visando investigar a influência da presença da matéria orgânica nos resultados das análises, foi realizado um experimento de adição e recuperação de $\mathrm{Cr}(\mathrm{VI})$ em duas porções de uma mesma amostra de solo, porém uma delas foi calcinada antes da adição da alíquota de $\operatorname{Cr}(\mathrm{VI})$, ou seja, toda a matéria orgânica dessa alíquota de solo foi eliminada. As adições de $\mathrm{Cr}(\mathrm{VI})$ às duas porções de solo foram efetuadas antes das amostragens das replicatas e as amostras foram homogeneizadas utilizando almofariz e pistilo e secas em estufa, conforme descrito anteriormente. Os teores de matéria orgânica e as recuperações de $\mathrm{Cr}(\mathrm{VI})$ de ambas as porções da amostra de solo estão apresentadas na Tabela 4.

Tabela 4. Teores de matéria orgânica e recuperação de $\mathrm{Cr}(\mathrm{VI})$ em amostras de solo não calcinada e calcinada

\begin{tabular}{lcc}
\hline Amostra & M. O. $\left(\mathrm{g} \mathrm{kg}^{-1}\right)$ & Recuperação de $\mathrm{Cr}(\mathrm{VI})(\%)$ \\
\hline $\mathrm{S}_{1}$ & $13,0 \pm 0,3$ & 44 \\
$\mathrm{~S}_{\text {lcalcinado. }}$ & $<1,0$ & 95
\end{tabular}

Os resultados representam valores médios e os respectivos desvios padrão $(\mathrm{n}=3)$.

No solo calcinado, com teor de matéria orgânica abaixo do limite de detecção, a recuperação de $\mathrm{Cr}(\mathrm{VI})$ foi próxima a 100\%. No entanto, para o solo contendo $13 \mathrm{~g} \mathrm{~kg}^{-1}$ de matéria orgânica, a recuperação foi de $44 \%$, ou seja, inferior à metade da concentração adicionada de $\mathrm{Cr}(\mathrm{VI})$. Esses dados confirmam que, realmente, a presença de matéria orgânica afeta de forma pronunciada a recuperação do $\mathrm{Cr}(\mathrm{VI})$, sendo essa redução imediata, considerando-se que as determinações foram realizadas logo após a secagem do solo, ou seja, cerca de 48 h. Deve-se salientar que o solo utilizado nesse experimento possui teor de matéria orgânica inferior ao solo contaminado.

Assim, podem-se atribuir os teores de $\mathrm{Cr}(\mathrm{VI})$ abaixo do limite de detecção apresentados pelas amostras de solo contaminadas com solução sulfocrômica residual à redução do $\mathrm{Cr}(\mathrm{VI})$ pela matéria orgânica do solo anteriormente ao processo de análise. 


\section{CONCLUSÕES}

A presença de matéria orgânica no solo avaliado foi capaz de promover a redução de $\mathrm{Cr}(\mathrm{VI})$ a $\mathrm{Cr}(\mathrm{III})$. Essa redução pode ter sido facilitada pelo meio ácido proporcionado pela presença de solução sulfocrômica, elevando o potencial redox do $\mathrm{Cr}(\mathrm{VI})$. Assim, pode-se supor que a contaminação do solo em estudo por $\mathrm{Cr}(\mathrm{VI})$ poderia ser mais drástica caso o ambiente apresentasse baixo teor de matéria orgânica ou pH's alcalinos, pois dessa forma o cromo tenderia a permanecer na forma $\mathrm{Cr}(\mathrm{VI})$.

\section{AGRADECIMENTOS}

Às instituições de fomento CAPES, CNPq e FAPESP pelo indispensável apoio e ao Dr. C. H. Kurihara, pesquisador da Embrapa Agropecuária Oeste pelo fornecimento das amostras de solo e preocupação com a qualidade ambiental.

\section{REFERÊNCIAS}

1. Barceloux, D. G.; J. Toxicol. Clin. Toxicol. 1999, 37, 173.

2. International Organization for Standardization; Method ISO 14235, Soil quality - Determination of organic carbon by sulphochromic oxidation, 1998.

3. Emsley, J.; The Elements, Oxford University Press: Cambridge, 1989.

4. Anderson, R. A.; J. Am. Coll. Nutr. 1998, 17, 548.

5. Shrivastava, R.; Upreti, R. K.; Seth, P. K.; Chaturvedi, U. C.; FEMS Immunol. Med. Microbiol. 2002, 34, 1.

6. Zachara, J. M.; Ainsworth, C. C.; Cowan, C. E.; Resch, C. T.; Soil Sci.
Soc. Am. J. 1989, 53, 418.

7. Rai, D.; Sass, B. M.; Moore, D. A.; Inorg. Chem. 1987, 26, 345.

8. Sass, B. M.; Rai, D.; Inorg. Chem. 1987, 26, 2228.

9. Azevedo, S. A.; Chasin, A. A. M.; Metais: gerenciamento da toxicidade, Ed. Atheneu: São Paulo, 2003.

10. Swedish Environmental Protection Agency; Guidelines for polluted soils, 2002.

11. Canadian Council of Ministers of the Environment; Canadian Environmental Quality Guidelines, Winnipeg, 2001.

12. Pettine, M.; Capri, S.; Anal. Chim. Acta 2005, 540, 231.

13. Decreto Ministerialen. 471; Gazzetta Ufficiale Supplemento Ordinario N. 293, 1999.

14. Eid, Z.; J. Environ. Sci. Health, Part A: Toxic/Hazard. Subst. Environ. Eng. 1996, 31, 227.

15. Palmer, C. D.; Wittbrodt, P. R.; Environ. Health Perspt. 1991, 92, 25.

16. Panichev, N.; Mandiwana, K.; Foukaridis, G.; Anal. Chim. Acta 2003, 491, 81.

17. Marczenko, Z.; Separation and spectrophotometric determination of elements, Ellis Horwood: Chichester, 1986.

18. Vieira, E. C.; Kamogawa, M. Y.; Lemos, S. G.; Nóbrega, J.A.; Nogueira, A. R. A.; R. Bras. Ci. Solo 2005, 29, 547.

19. Kotás, J.; Stasicka, Z.; Environ. Pollut. 2000, 107, 263.

20. Companhia de Tecnologia de Saneamento Ambiental - CETESB; Decisão de diretoria $N^{\circ}$ 195-2005- E, de 23 de novembro de 2005.

21. Bartlett, R. J.; Kimble, J. M.; J. Environ. Qual. 1976, 3, 379.

22. James, B. R.; Bartlett, R. J. ; J. Environ. Qual. 1983, 12, 177.

23. Kozyuh, N.; Sytupar, J.; Gorenc, B.; Environ. Sci. Technol. 2000, 34, 112. 